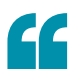

Knockdown of SYK using short hairpin RNAs indicated that SYK is required for retinoblastoma cell survival.

\title{
RETINOBLASTOMA
}

\section{Epigenetic outcome}

Retinoblastoma is a childhood cancer that arises owing to the inactivation of both copies of the tumour suppressor $R B 1$. A recent paper published in Nature shows that, aside from this genetic change, few genetic alterations are evident and instead epigenetic changes are paramount.

Whole-genome sequencing was carried out on four samples of retinoblastoma from the St Jude Children's Hospital-Washington University Pediatric Cancer Genome Project and the paired normal samples. Interestingly, despite the association of $R B 1$ loss with aneuploidy and chromosomal instability, the four genomes sequenced in this paper were relatively stable. On the basis of these findings, the authors switched their attention to epigenetic alterations. By comparing chromatin immunoprecipitation data, DNA methylation assays and gene expression assays from retinoblastoma with control human retinal samples, 104 genes were shown to have differential expression. One of these, spleen tyrosine kinase (SYK), which is involved in regulating the immune response, had alterations in histone modifications associated with active gene transcription. Moreover, $100 \%$ of 82 samples of human retinoblastoma showed increased expression of SYK, as determined by immunohistochemistry. Knockdown of SYK using short hairpin RNAs indicated that SYK is required for retinoblastoma cell survival. Similar results were seen when small-molecule inhibitors of SYK were used in vitro, and addition of a SYK inhibitor prolonged the survival of mice with retinoblastoma orthotopic xenografts treated with topotecan hydrochloride.

These findings indicate that epigenetic mechanisms that contribute to retinoblastoma might be viable avenues for therapy.

Nicola McCarthy

ORIGINAL RESEARCH PAPER Zhang, J. et al.

A novel retinoblastoma therapy from genetic and epigenetic analyses. Nature 11 Jan 2012 (doi:10.1038/nature10733)

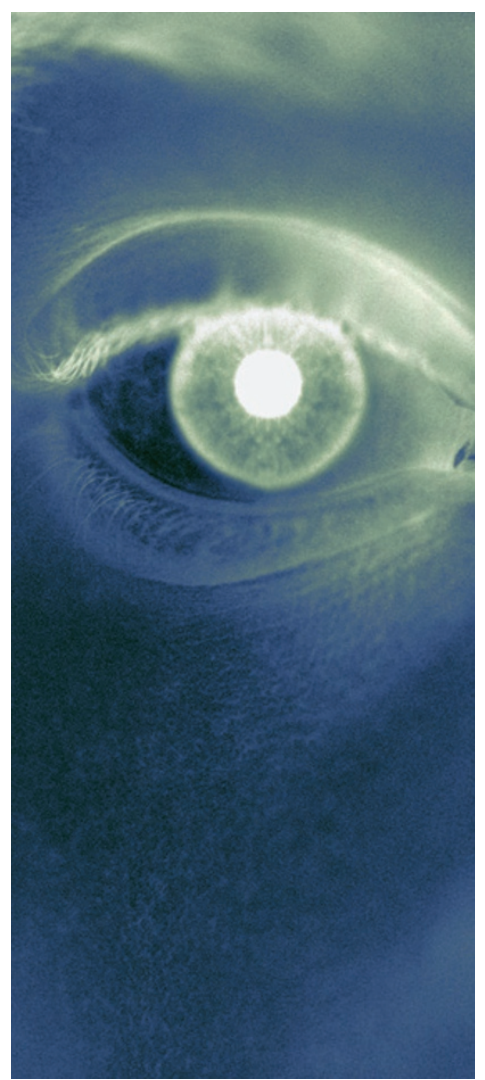

